\title{
Clinical significance of ZNF750 gene expression, a novel tumor suppressor gene, in esophageal squamous cell carcinoma
}

\author{
SHO NAMBARA $^{1}$, TAKAAKI MASUDA ${ }^{1}$, TARO TOBO ${ }^{2}$, SHINYA KIDOGAMI $^{1}$, HISATERU KOMATSU ${ }^{1}$, \\ KEISHI SUGIMACHI ${ }^{1}$, HIROSHI SAEKI ${ }^{3}$, EIJI OKI ${ }^{3}$, YOSHIHIKO MAEHARA ${ }^{3}$ and KOSHI MIMORI ${ }^{1}$ \\ Departments of ${ }^{1}$ Surgery and ${ }^{2}$ Pathology, Kyushu University Beppu Hospital, Beppu, Oita 874-0838; \\ ${ }^{3}$ Department of Surgery and Science, Graduate School of Medical Sciences, \\ Kyushu University, Fukuoka, Fukuoka 812-8582, Japan
}

Received October 31, 2016; Accepted March 28, 2017

DOI: $10.3892 / \mathrm{ol} .2017 .6341$

\begin{abstract}
The present authors previously identified a novel candidate tumor suppressor gene, zinc finger protein 750 (ZNF750), in esophageal squamous cell carcinoma (ESCC) (1). The present study aimed to clarify the clinical significance of ZNF750 expression in ESCC. The association between ZNF750 DNA mutation status and the mRNA expression was examined by whole exome sequence analysis and quantitative reverse transcription polymerase chain reaction (RT-qPCR). The expression of ZNF750 in 76 patients with ESCC (Kyushu University Beppu Hospital) was measured using immunohistochemistry and RT-qPCR. Using this dataset, the association between ZNF750 mRNA expression and clinicopathological factors was examined. Additionally, survival analysis was performed using datasets from the Kyushu University Beppu Hospital and The Cancer Genome Atlas (TCGA). The biological effects of ZNF750 expression were explored using gene set enrichment analysis (GSEA) and were validated using datasets from the Cancer Cell Line Encyclopedia (CCLE) and the Kyushu University Beppu Hospital. ZNF750 expression analyses demonstrated that ZNF750 mRNA expression was lower in patients with the DNA mutations compared with those without the mutations $(\mathrm{P}<0.05)$, and ZNF750 expression was downregulated in tumor tissues compared with normal tissues $(\mathrm{P}<0.00005)$. In the clinicopathological analysis, the low ZNF750 expression group exhibited a higher incidence of undifferentiated histology $(\mathrm{P}<0.05)$ compared with the high expression group. The low ZNF750 expression group exhibited a poorer prognosis in the Kyushu and TCGA datasets $(\mathrm{P}<0.0005$ and $\mathrm{P}<0.05$, respectively). GSEA indicated that
\end{abstract}

Correspondence to: Professor Koshi Mimori, Department of Surgery, Kyushu University Beppu Hospital, 4546 Tsurumihara, Beppu, Oita 874-0838, Japan

E-mail:kmimori@beppu.kyushu-u.ac.jp

Key words: zinc finger protein 750, esophageal squamous cell carcinoma, tumor suppressor gene, poor prognostic biomarker, epithelial differentiation
ZNF750 expression was significantly correlated with epithelial differentiation in ESCC. This was confirmed using the datasets from CCLE and the Kyushu University Beppu Hospital by analyzing the levels of small proline rich protein 1A mRNA, an epithelial differentiation-associated gene. In conclusion, the results of the present study suggested that ZNF750 serves a role as a tumor suppressor; potentially via regulating epithelial differentiation and that it may be a promising biomarker of poor outcomes in ESCC.

\section{Introduction}

Esophageal squamous cell carcinoma (ESCC) is the most common histological type of esophageal cancer worldwide (2). ESCC tends to have a poor prognosis due to high rates of lymph node metastasis and invasion into surrounding organs (2). ESCC has been identified to harbor frequent somatic mutations in tumor protein 53 (3), Notch homolog 1, tumor protein P53 (3) and phosphatidylinositol-4,5-biphosphate 3-kinase catalytic subunit $\alpha$ (4). However, previous cohorts used to investigate the genomic abnormalities of ESCC were too small to encompass the full range of genomic abnormalities $(3,4)$. The present authors have recently performed whole-exome sequence (WES) analysis on 144 Japanese patients with ESCC, and identified a previously uncharacterized mutant gene, zinc finger protein 750 (ZNF750) (1), which is an essential regulator of epithelial proliferation and differentiation (5-7).

In general, in order to identify a sporadic tumor suppressor gene, studies should focus on the high frequency aberrant genomic loci in familial cancer syndromes. As such, it was identified that ZNF750 is located at chromosome 17q25, the same location for the gene responsible for hereditary tylosis esophageal cancer, a familial esophageal cancer $(8,9)$.

In a previous study by the present authors, mutations in ZNF750 were identified in $16.7 \%$ of ESCC tumors and ZNF750 was identified to be the fifth most frequent mutated genes. Additionally, $87.5 \%$ of mutations in ZNF750 led to decreased mRNA expression (1). Similarly, Lin et al (10) and Zhang et al (11) also revealed inactivating mutations (70.0 and $64.0 \%$ of mutations in each study, respectively) in ZNF750 by WES analysis of ESCC and demonstrated that the depletion of ZNF750 in ESCC cell lines promoted proliferation and 
invasiveness. These results suggested that ZNF750 serves a role as a tumor suppressor in ESCC. However to the best our knowledge, no study has investigated whether aberrant ZNF750 expression affected clinical outcomes in ESCC. Elucidation of the clinical significance of ZNF750 expression may clarify whether ZNF750 may be targeted in clinical therapy. Therefore, the present study aimed to clarify the clinicopathological and prognostic significance of ZNF750 expression in ESCC using two independent ESCC datasets.

\section{Materials and methods}

ESCC patients and sample collection. A total of 76 primary ESCC samples and 76 paired normal tissues were obtained from 76 patients who underwent surgery at Kyushu University Beppu Hospital (Beppu, Japan) and affiliated hospitals [Iwate Medical University (Iwate, Japan), Kurume University School of Medicine (Kurume, Japan), and Kagoshima University Graduate School of Medical and Dental Sciences (Kagoshima, Japan)] from January, 1998 to December, 2012 (Kyushu dataset). The samples were taken as a part of routine examination. The patient group comprised 67 males and 8 females (the gender of one patient was not available). The mean age of the patients was 64.7 years (range, 40.0-79.0). All patients had a histological diagnosis of ESCC and were closely followed at 3-month intervals. The median follow-up period was 3.9 years. All patients were treated in accordance with the Japan Esophageal Society Guidelines for the treatment of esophageal cancer (12). Written informed consent was obtained from all patients, and the Institutional Review Board of Kyushu University (Fukuoka, Japan) approved the present study. Sample collection was performed as previously described (13). Data on patient age, sex, histology, tumor depth of invasion, lymph node metastasis, lymphatic invasion, venous invasion and distant metastasis were obtained from the clinical and pathological records. A total of two patients who exhibited distant metastasis were included in the present study. Of these patients, one exhibited distant lymph node metastasis and the other liver metastasis.

ZNF750 mutation. ZNF750 mutation status data were obtained from WES analysis that the authors of the present study previously performed (1). RNA from 28 patients of the 144 used in the previous study was available for quantitative reverse transcription polymerase chain reaction (RT-qPCR) analysis.

Total RNA extraction and RT. Total RNA from tissues was extracted by a modified AGPC method using ISOGEN (Nippon Gene Co., Ltd., Tokyo, Japan), in which the aqueous phase extraction step by chloroform is unrequired (14). RT was performed using $8 \mu \mathrm{g}$ of total RNA with Moloney Murine leukemia virus reverse transcriptase according to the manufacturer's protocol (Invitrogen; Thermo Fisher Scientific, Inc., Waltham, MA, USA).

Quantitative (q)PCR. qPCR reactions were performed using a LightCycler 480 probes master kit (Roche Diagnostics, Indianapolis, IN, USA) and repeated three times as previously described (15). The following primers were used: ZNF750; sense primer, 5'-GAACAGGTACTGCTTCCTGAGC-3' and antisense primer, 5'GAGAGCCTCCGTCATCTGG-3'; small proline rich protein 1A (SPRRIA); sense primer, 5'-TCC ACCCCAGGAACCATGC-3' and antisense primer, 5'-CTT GGTCTTCTGCTGGGCTG-3'; glyceraldehyde-3-phosphate dehydrogenase $(G A P D H)$; sense primer, 5'-TTGGTATCG TGGAAGGACTCTA-3' and antisense primer, 5'-TGTCAT ATTTGGCAGGTT-3. The expression levels of ZNF750 and SPRRIA were normalized by GAPDH as an internal control. The expression levels were calculated as the values relative to the expression level of the cDNA using Human Universal Reference Total RNA with the calibration curve method (Clontech Laboratories, Inc., Mountainview, CA, USA) (16).

Immunohistochemical analysis. Immunohistochemistry of ZNF750 in 5 randomly selected representative ESCC cases was performed on formalin-fixed, paraffin-embedded tissues as previously described (15). A polyclonal rabbit anti-ZNF750 antibody (cat. no., HPA023012, Sigma-Aldrich; Merck KGaA, Darmstadt, Germany; dilution, 1:100) was used as the primary antibody. Tumor histology was independently reviewed by an experienced pathologist.

The Cancer Genome Atlas (TCGA) data analysis. Paired RNA sequencing and survival data of 86 available patients with ESCC were obtained from TCGA (http://cancergenome. nih.gov/). ZNF750 mRNA expression and survival data were extracted from this reference.

Gene set enrichment analysis (GSEA). The correlations between ZNF750 expression and previously annotated gene expression signatures were analyzed by applying GSEA (17). ESCC expression profiles from the National Center for Biotechnology Information (NCBI) gene expression omnibus database (NCBI accession no. GSE2533) were acquired, and analyzed using GSEA, as previously described (18). Gene sets of ZNF750 targets were extracted from C2 curated gene sets in the Broad Institute database (http://www.broadinstitute .org/gsea/msigdb/collections.jsp).

Cancer Cell Line Encyclopedia (CCLE) data analysis. Normalized mRNA expression data of 22 available ESCC cell lines were obtained from the CCLE dataset (http://www .broadinstitute.org/ccle/home). ZNF750 and SPRR1A mRNA expression data were extracted from this reference.

Statistical analysis. For continuous variables, statistical analyses were performed using Student's t-test for comparisons of ZNF750 mRNA expression according to the DNA mutation status (un-paired t-test) and between corresponding tumor and normal tissues (paired t-test). The degree of linearity was estimated by Pearson's correlation coefficient. The association between ZNF750 mRNA expression and clinicopathological factors was estimated by the $\chi^{2}$ test with Yates' correction. Overall survival was estimated using the Kaplan-Meier method, and survival curves were compared using log-rank tests. Univariate and multivariate analyses were used to determine patient prognosis and were performed by Cox regression analysis with a backward stepwise model. Based on the levels of ZNF750 mRNA expression in the Kyushu and the TCGA datasets, patients were divided into two groups using 

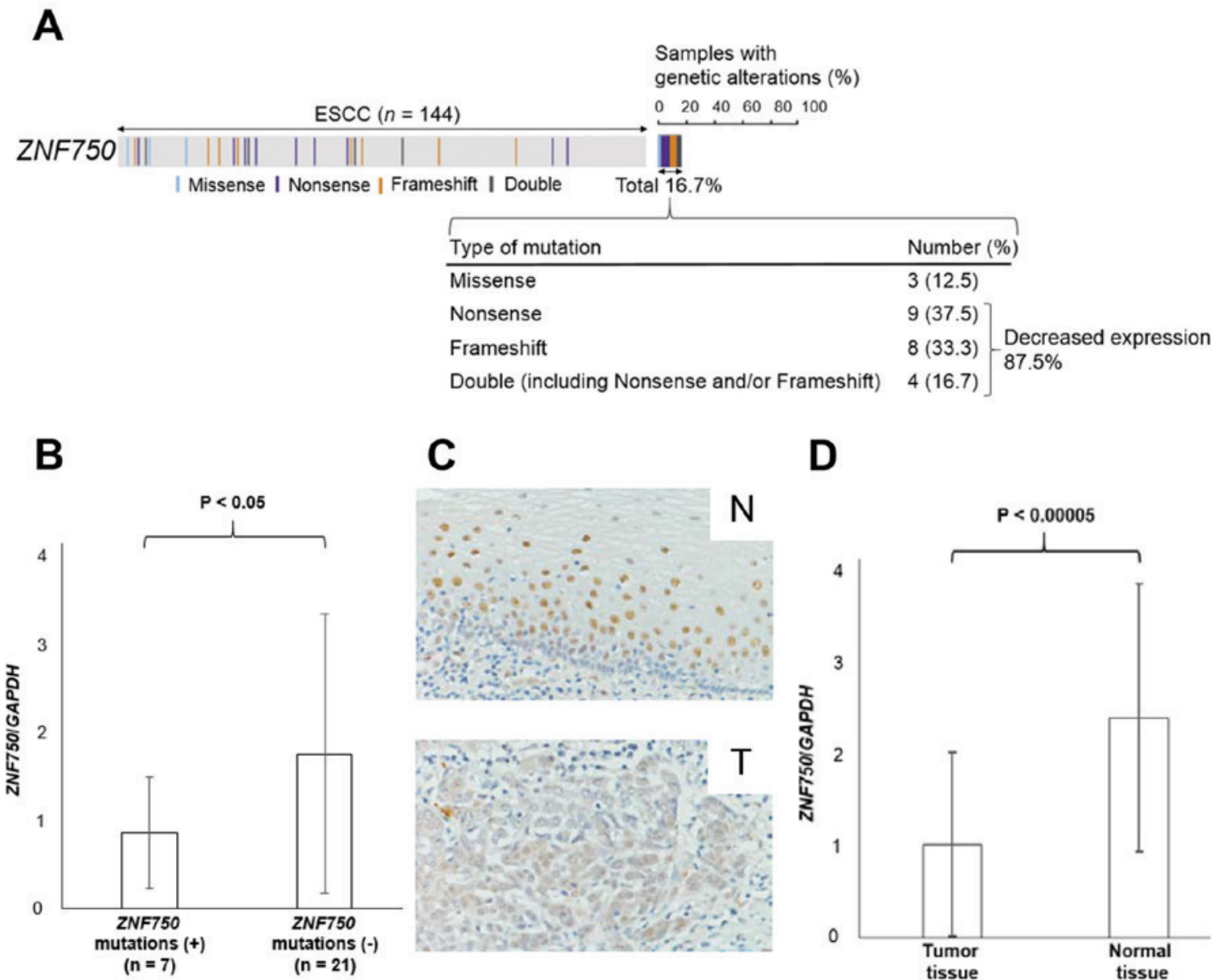

Figure 1. ZNF750 expression in ESCC. (A) Overview of genetic alterations of ZNF750 across 144 patients with ESCC from a previous report by the present authors (4). (B) ZNF750 mRNA expression in ESCC according to DNA mutation status. (P<0.05; Student's t-test). (C) Immunohistochemical staining of ZNF750 expression in representative samples of ESCC. Original magnification, $\mathrm{x} 400$. (D) ZNF750 mRNA expression in ESCC assessed by quantitative reverse transcription polymerase chain reaction. ( $\mathrm{P}<0.00005$; Student's t-test). N, normal tissue; T, Tumor tissue. ESCC, esophageal squamous cell carcinoma; GAPDH, glyceraldehyde-3-phosphate dehydrogenase; ZNF750, zinc finger protein 750.

the minimum P-value approach, a comprehensive method that determines the optimal risk separation cutoff point in continuous gene expression measurement (19). The cutoff values for high and low ZNF750 expression groups were 8.34 (ZNF750/GAPDH expression) in the Kyushu dataset and 2.75 ( $\log 2$ expression) in the TCGA dataset. All tests were analyzed by using the JMP v.11 software (JMP, Cary, NC, USA). Depth of tumor invasion was classified according to the 7th edition of the International Union against Cancer TNM classification system (20). Histological grade of the tumor was classified according to the 11th edition of the Japanese Classification of Esophageal Cancer (21).

\section{Results}

Downregulation of ZNF750 expression in ESCC. It was revealed that $16.7 \%$ of patients with ESCC exhibited mutations in the ZNF750 gene (4) (Fig. 1A). The majority of the mutations (87.5\%) were nonsense and/or frameshift mutations that caused reduced expression of ZNF750 (Fig. 1A). As expected, ZNF750 mRNA expression was significantly lower in patients with $Z N F 750$ nonsense and/or frameshift mutations $(n=7)$ compared with those without mutations $(n=21)(P<0.05$; Fig. 1B). Consistent with these data, the immunohistochemical analysis revealed that ZNF750 was stained less intensely in the nuclei of ESCC tumor cells compared with those of normal esophageal epithelial cells in 4 of 5 (80.0\%) patients (Fig. 1C). Additionally, RT-qPCR analysis also demonstrated that ZNF750 mRNA expression in tumor tissues was significantly lower compared with paired normal tissues $(\mathrm{P}<0.00005)$ (Fig. 1D). In fact, ZNF750 mRNA expression levels in tumor tissues were lower compared with normal tissues in $78.9 \%$ of 76 patients with ESCC.

Clinicopathological significance of ZNF750 mRNA expression in ESCC. The association between ZNF750 mRNA expression levels in tumor tissues and clinicopathological factors in ESCC is summarized in Table I. The low ZNF750 expression group $(n=37)$ exhibited a higher frequency of tissues showing poorly differentiated histology $(\mathrm{P}<0.05)$ compared with the high expression group $(\mathrm{n}=39)$. No significant associations between ZNF750 mRNA expression and age, sex, depth of invasion, lymph node metastasis, lymphatic invasion, venous invasion or distant metastasis were observed.

Prognostic significance of ZNF750 mRNA expression in $E S C C$. The overall survival rate of patients in the low ZNF750 expression group was significantly lower compared with 
Table I. Association between ZNF750 mRNA expression of tumor tissues and clinicopathological factors in ESCC.

\begin{tabular}{|c|c|c|c|}
\hline Factors & $\begin{array}{c}\text { High } Z N F 750 \\
\text { expression }(n=39) n,(\%)\end{array}$ & $\begin{array}{c}\text { Low } Z N F 750 \\
\text { expression }(n=37) n,(\%)\end{array}$ & P-value \\
\hline$<65$ & $20(51.3)$ & $18(48.6)$ & NS \\
\hline$\geq 65$ & $19(48.7)$ & $19(51.4)$ & \\
\hline Male & $35(89.7)$ & $32(86.5)$ & NS \\
\hline Female & $4(10.3)$ & $4(10.8)$ & \\
\hline NA & $0(0)$ & $1(2.7)$ & \\
\hline Well & $17(43.6)$ & $7(18.9)$ & $<0.05$ \\
\hline Moderate/poor & $17(43.6)$ & $22(59.5)$ & \\
\hline NA & $5(12.8)$ & $8(21.6)$ & \\
\hline$\leq$ submucosa $(\mathrm{T} 1)$ & $8(20.5)$ & $2(5.4)$ & NS \\
\hline$\geq$ muscularis propria $(\mathrm{T} 2-\mathrm{T} 4)$ & $31(79.5)$ & $35(94.6)$ & \\
\hline Absent & $12(30.8)$ & $10(27.0)$ & NS \\
\hline Present & $27(69.2)$ & $27(73.0)$ & \\
\hline Absent & $8(20.5)$ & $3(8.1)$ & NS \\
\hline Present & $31(79.5)$ & $33(89.2)$ & \\
\hline NA & $0(0)$ & $1(2.7)$ & \\
\hline Absent & $8(20.5)$ & $2(5.4)$ & NS \\
\hline Present & $31(79.5)$ & 34 (91.9) & \\
\hline NA & $0(0)$ & $1(2.7)$ & \\
\hline Absent & $38(97.4)$ & $36(97.3)$ & NS \\
\hline Present & $1(2.6)$ & $1(2.7)$ & \\
\hline
\end{tabular}

NA, not available; NS, not significant.
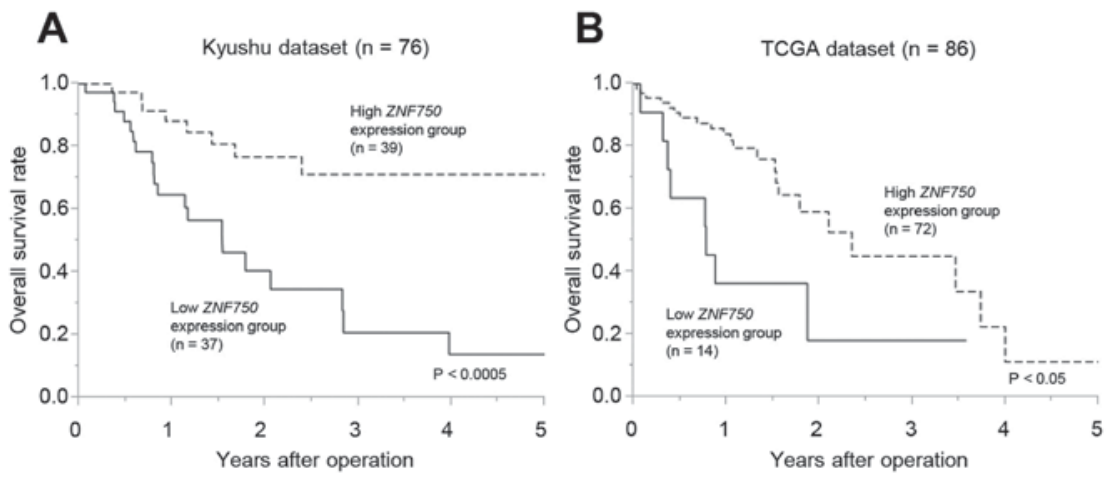

Figure 2. Prognostic significance of ZNF750 mRNA expression in ESCC. Overall survival rate of patients with ESCC according to ZNF750 mRNA expression in tumor tissues. (A) Kyushu dataset $(\mathrm{P}<0.0005)$. (B) TCGA dataset $(\mathrm{P}<0.05)$. TCGA, The Cancer Genome Atlas; ESCC, Esophageal squamous cell carcinoma; ZNF750, zinc finger protein 750 .

the high expression group in the Kyushu dataset $(\mathrm{P}<0.0005$; Fig. 2A) and the TCGA dataset $(\mathrm{P}<0.05)$ (Fig. 2B). In the univariate analysis, higher $\mathrm{T}$ factor (T2-4), lymph node metastasis, lymphovascular invasion and low ZNF750 mRNA expression were significantly associated with a lower overall survival rate (Table II). The multivariate analysis demonstrated that lymphatic invasion $(\mathrm{P}<0.05)$ and low ZNF750 mRNA expression $(\mathrm{P}<0.05)$ were independent poor prognostic factors in ESCC (Table II).

Correlation between ZNF750 mRNA expression levels and epithelial differentiation genes in ESCC.In order to validate the clinicopathological analysis of ZNF750 in ESCC, GSEA was applied to patients with ESCC (NCBI accession no. GSE2533). GSEA demonstrated that ZNF750 expression was significantly correlated with epithelial differentiation genes (Fig. 3A), which suggests that $Z N F 750$ serves a role in epithelial differentiation in the development of ESCC. Among those genes, SPRRIA, an early epithelial differentiation marker (22), was ranked at the upper level of epithelial differentiation gene signature. The analysis confirmed that ZNF750 mRNA expression was positively correlated with SPRRIA mRNA expression using the CCLE dataset $(\mathrm{R}=0.71$; Fig. 3B) and the Kyushu dataset ( $\mathrm{R}=0.56$; Fig. 3C). 
Table II. Univariate and multivariate analysis of clinicopathological factors affecting overall survival rate.

\begin{tabular}{|c|c|c|c|c|c|c|}
\hline \multirow[b]{2}{*}{ Factors } & \multicolumn{3}{|c|}{ Univariate analysis } & \multicolumn{3}{|c|}{ Multivariate analysis } \\
\hline & RR & $95 \% \mathrm{CI}$ & P-value & RR & $95 \% \mathrm{CI}$ & P-value \\
\hline Age $(\geq 65 /<65)$ & 0.93 & $0.46-1.91$ & NS & 1.68 & $0.58-5.15$ & NS \\
\hline Sex (male/female) & 1.19 & $0.45-4.13$ & NS & 1.21 & $0.26-7.54$ & NS \\
\hline Histology grade (well/moderate and poor) & 0.62 & $0.24-1.51$ & NS & 1.89 & $0.59-5.66$ & NS \\
\hline $\mathrm{T}$ factor $(\mathrm{T} 1 / \mathrm{T} 2-4)$ & 0.31 & $0.07-0.94$ & $<0.05$ & 0.22 & $0.03-1.11$ & NS \\
\hline Lymph node metastasis (negative/positive) & 0.43 & $0.17-0.98$ & $<0.05$ & 0.53 & $0.11-1.92$ & NS \\
\hline Lymphatic invasion (negative/positive) & $5.42 \times 10^{-10}$ & $0.34-2.96$ & $<0.005$ & $3.37 \times 10^{-10}$ & $0.47-2.11$ & $<0.05$ \\
\hline Venous invasion (negative/positive) & $6.02 \times 10^{-10}$ & $0.48-2.08$ & $<0.05$ & $1.67 \times 10^{-9}$ & $0.37-2.68$ & NS \\
\hline Distant metastasis (negative/positive) & 5.84 & $0.92-20.79$ & NS & 11.01 & $0.51-23.34$ & NS \\
\hline ZNF750 mRNA expression (high/low) & 3.83 & $1.82-8.59$ & $<0.0005$ & 3.83 & $1.22-13.44$ & $<0.05$ \\
\hline
\end{tabular}

RR, relative risk; CI, confidence interval; NS, not significant; T, Depth of tumor invasion.

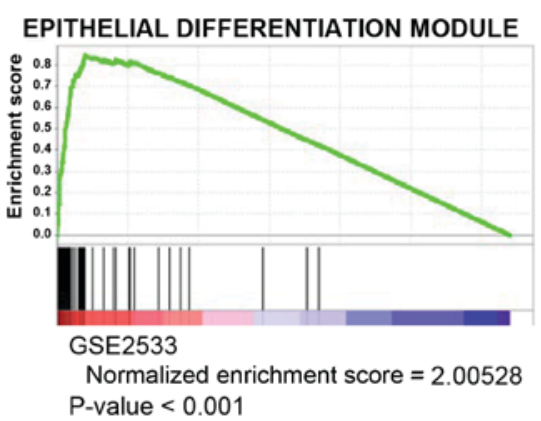

B

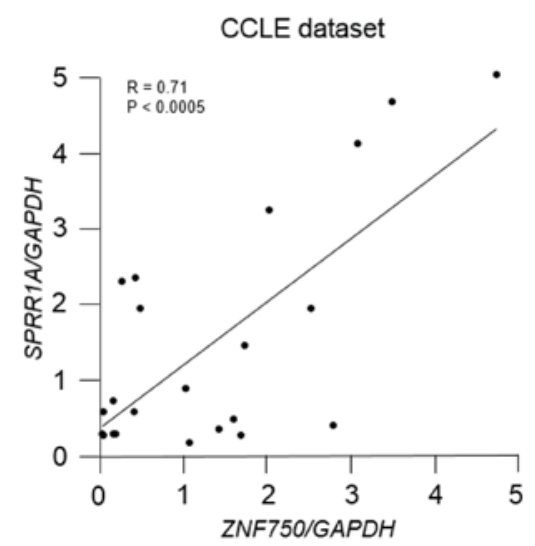

C

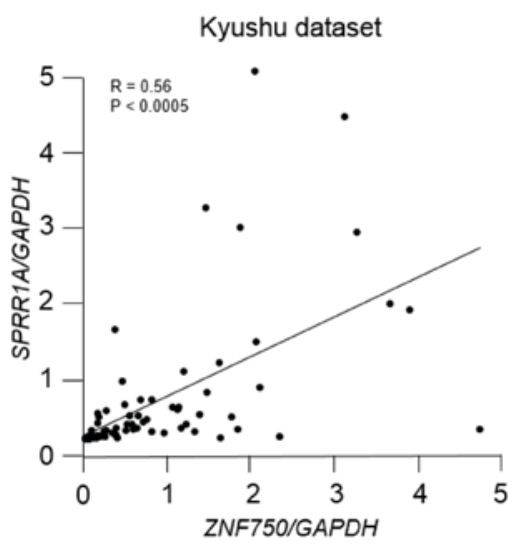

Figure 3. Correlation between ZNF750 mRNA expression levels and epithelial differentiation genes in ESCC. (A) Gene set enrichment analysis of interrelated ZNF750 expression and epithelial differentiation module using reference gene sets in the ESCC dataset. (B) Correlation between ZNF750 and SPRR1A expression in the CCLE dataset. (C) Correlation between ZNF750 and SPRR1A expression in the Kyushu dataset. ESCC, esophageal squamous cell carcinoma; GAPDH, glyceraldehyde-3-phosphate dehydrogenase; SPRR1A, small proline rich protein 1A; ZNF750, zinc finger protein 750; CCLE, Cancer Cell Line Encyclopedia.

\section{Discussion}

In the present study, the clinicopathological and prognostic significance of ZNF750 expression, a novel candidate tumor suppressor gene previously described by the present authors (4), was assessed. In order to assess the prognostic significance of ZNF750 expression, two independent ESCC datasets were used. In addition, an association between ZNF750 expression and epithelial differentiation in ESCC was identified. The data of the present study suggested that the downregulation of ZNF750 expression due to inactivating mutations (nonsense/frameshift mutations) induced the development or aggressiveness of ESCC, potentially via dysregulation of esophageal epithelial differentiation. 
Although other factors such as hyper-methylation or regulation by microRNA may also downregulate the expression of ZNF750, to the best of our knowledge, this is the first study to explore the clinical significance of ZNF750 expression in ESCC.

The present clinicopathological study revealed that the tissues from the low ZNF750 expression group exhibited a higher frequency of poorly differentiated histology compared with the high expression group. Previously, it was suggested that ZNF750 function was associated with epithelial differentiation $(5-7,10,11)$. These data suggested that a low expression of ZNF750 is associated with tumor aggressiveness in ESCC.

Additionally, it was demonstrated that ZNF750 expression was reduced in tumor tissues, and that low expression of ZNF750 was an independent poor prognostic factor in ESCC. In a previous study by the present authors (4), it was also demonstrated that the majority of the ZNF750 mutations were accompanied by loss of heterozygosity. These data strongly support the hypothesis that ZNF750 is a tumor suppressor gene, as previously identified by WES analysis. In addition, low ZNF750 expression levels in tumor tissues suggest that it is a promising biomarker capable of predicting poor outcomes for patients with ESCC.

It has been reported that ZNF750 controls epithelial homeostasis by repressing proliferation genes and inducing differentiation genes (5-7). Previous studies indicated that mutations in ZNF750 were pathogenic and disrupt epithelial homeostasis, as observed in psoriasis (23). The present study suggested that ZNF750 expression was significantly correlated with epithelial differentiation genes such as SPRRIA, an early epithelial differentiation marker, in ESCC. The downregulation of ZNF750 expression in ESCC may also disrupt epithelial homeostasis, followed by the development of ESCC, according to Tetreault et al (24), who demonstrated that the downregulation of Kruppel like factor 4, an important epithelial differentiation gene, induced malignant transformation of esophageal epithelium. However, the function of the ZNF750 gene in ESCC has not been fully elucidated. Additional large clinical studies and biological experiments for ZNF750 in ESCC are required to clarify this.

In conclusion, ZNF750 expression was low in ESCC tumor tissues, and its reduction was associated with poor differentiation. In addition, low ZNF750 expression was an independent poor prognostic factor in ESCC. These data provide evidence that ZNF750 is a novel tumor suppressor gene, and may be a therapeutic target and useful biomarker of poor clinical outcome in patients with ESCC.

\section{Acknowledgements}

The authors would like to thank Ms. M. Oshiumi, Mr. M. Utou, Mrs. K. Oda, Ms. M. Kasagi, Mrs. S. Sakuma, Mrs. N. Mishima and Ms. T. Kawano (Departments of Surgery and Pathology, Kyushu University Beppu Hospital, Beppu, Japan) for their excellent technical assistance. The present study was supported by the following grants and foundations: Grants-in-Aid for Scientific Research of MEXT (grant nos. 26461980, 26293303, 26670608, 26861003 and 26271401); Japan Agency for Medical Research and development, AMED (grant no. P14009); The OITA Cancer Research Foundation 2015; Daiwa Securities Health Foundation.

\section{References}

1. Sawada G, Niida A, Uchi R, Hirata H, Shimamura T, Suzuki Y, Shiraishi Y, Chiba K, Imoto S, Takahashi Y, et al: Genomic landscape of esophageal squamous cell carcinoma in a Japanese population. Gastroenterology 150: 1171-1182, 2016.

2. Pennathur A, Gibson MK, Jobe BA and Luketich JD: Oesophageal carcinoma. Lancet 381: 400-412, 2013.

3. Agrawal N, Jiao Y, Bettegowda C, Hutfless SM, Wang Y, David S, Cheng Y, Twaddell WS, Latt NL, Shin EJ, et al: Comparative genomic analysis of esophageal adenocarcinoma and squamous cell carcinoma. Cancer Discov 2: 899-905, 2012.

4. Shigaki H, Baba Y, Watanabe M, Murata A, Ishimoto T, Iwatsuki M, Iwagami S, Nosho K and Baba H: PIK3CA mutation is associated with a favorable prognosis among patients with curatively resected esophageal squamous cell carcinoma. Clin Cancer Res 19: 2451-2459, 2013.

5. Cohen I, Birnbaum RY, Leibson K, Taube R, Sivan S and Birk OS: ZNF750 is expressed in differentiated keratinocytes and regulates epidermal late differentiation genes. PLoS One 7: e42628, 2012.

6. Sen GL, Boxer LD, Webster DE, Bussat RT, Qu K, Zarnegar BJ, Johnston D, Siprashvili Z and Khavari PA: ZNF750 is a p63 target gene that induces KLF4 to drive terminal epidermal differentiation. Dev Cell 22: 669-677, 2012.

7. Boxer LD, Barajas B, Tao S, Zhang J and Khavari PA: ZNF750 interacts with KLF4 and RCOR1, KDM1A, and CTBP1/2 chromatin regulators to repress epidermal progenitor genes and induce differentiation genes. Genes Dev 28: 2013-2026, 2014

8. Kelsell DP, Risk JM, Leigh IM, Stevens HP, Ellis A, Hennies HC, Reis A, Weissenbach J, Bishop DT, Spurr NK and Field JK: Close mapping of the focal non-epidermolytic palmoplantar keratoderma (PPK) locus associated with oesophageal cancer (TOC). Hum Mol Genet 5: 857-860, 1996.

9. Blaydon DC, Etheridge SL, Risk JM, Hennies HC, Gay LJ, Carroll R, Plagnol V, McRonald FE, Stevens HP, Spurr NK, et al: RHBDF2 mutations are associated with tylosis, a familial esophageal cancer syndrome. Am J Hum Genet 90: 340-346, 2012.

10. Lin DC, Hao JJ, Nagata Y, Xu L, Shang L, Meng X, Sato Y, Okuno Y, Varela AM, Ding LW, et al: Genomic and molecular characterization of esophageal squamous cell carcinoma. Nat Genet 46: 467-473, 2014.

11. Zhang L, Zhou Y, Cheng C, Cui H, Cheng L, Kong P, Wang J, Li Y, Chen W, Song B, et al: Genomic analyses reveal mutational signatures and frequently altered genes in esophageal squamous cell carcinoma. Am J Hum Genet 96: 597-611, 2015.

12. Kuwano H, Nishimura Y, Oyama T, Kato H, Kitagawa Y, Kusano M, Shimada H, Takiuchi H, Toh Y, Doki Y, et al: Guidelines for diagnosis and treatment of carcinoma of the esophagus april 2012 edited by the Japan esophageal society. Esophagus 12: 1-30, 2015.

13. Hirata H, Sugimachi K, Komatsu H, Ueda M, Masuda T, Uchi R, Sakimura S, Nambara S, Saito T, Shinden Y, et al: Decreased expression of fructose-1,6-bisphosphatase associates with glucose metabolism and tumor progression in hepatocellular carcinoma. Cancer Res 76: 3265-3276, 2016.

14. Chomczynski P and Mackey K: Short technical reports. Modification of the TRI reagent procedure for isolation of RNA from polysaccharide- and proteoglycan-rich sources. Biotechniques 19: 942-945, 1995.

15. Ueda M, Iguchi T, Nambara S, Saito T, Komatsu H, Sakimura S, Hirata H, Uchi R, Takano Y, Shinden Y, et al: Overexpression of transcription termination factor 1 is associated with a poor prognosis in patients with colorectal cancer. Ann Surg Oncol 22 (Suppl 3): S1490-S1498, 2015.

16. Sørensen BS, Schmidt H, von der Maase H, Straten PT and Nexø E: Quantification of melanoma cell-specific MART-1 mRNA in peripheral blood by a calibrated competitive reverse transcription-PCR. Clin Chem 46: 1923-1928, 2000.

17. Subramanian A, Tamayo P, Mootha VK, Mukherjee S, Ebert BL, Gillette MA, Paulovich A, Pomeroy SL, Golub TR, Lander ES and Mesirov JP: Gene set enrichment analysis: A knowledge-based approach for interpreting genome-wide expression profiles. Proc Natl Acad Sci USA 102: 15545-15550, 2005.

18. Wei G, Luo H, Sun Y, Li J, Tian L, Liu W, Liu L, Luo J, He J and Chen R: Transcriptome profiling of esophageal squamous cell carcinoma reveals a long noncoding RNA acting as a tumor suppressor. Oncotarget 6: 17065-17080, 2015. 
19. Mizuno H, Kitada K, Nakai K and Sarai A: PrognoScan: A new database for meta-analysis of the prognostic value of genes. BMC Med Genomics 2: 18, 2009.

20. Yamasaki M, Miyata H, Miyazaki Y, Takahashi T, Kurokawa Y, Nakajima K, Takiguchi S, Mori M and Doki Y: Evaluation of the nodal status in the 7th edition of the UICC-TNM classification for esophageal squamous cell carcinoma: Proposed modifications for improved survival stratification: Impact of lymph node metastases on overall survival after esophagectomy. Ann Surg Oncol 21: 2850-2856, 2014.

21. Japan Esophageal Society: Japanese classification of esophageal cancer, 11th edition: Part I. Esophagus 14: 1-36, 2017.
22. Sark MW, Fischer DF, de Meijer E, van de Putte P and Backendorf C: AP-1 and ets transcription factors regulate the expression of the human SPRR1A keratinocyte terminal differentiation marker. J Biol Chem 273: 24683-24692, 1998.

23. Yang CF, Hwu WL, Yang LC, Chung WH, Chien YH, Hung CF, Chen HC, Tsai PJ, Fann CS, Liao F and Chen YT: A promoter sequence variant of ZNF750 is linked with familial psoriasis. J Invest Dermatol 128: 1662-1668, 2008.

24. Tetreault MP, Yang Y, Travis J, Yu QC, Klein-Szanto A, Tobias JW and Katz JP: Esophageal squamous cell dysplasia and delayed differentiation with deletion of Krüppel-like factor 4 in murine esophagus. Gastroenterology 139: 171-181.e9, 2010. 